\title{
Article
}

\section{On a Boundary Value Problem of Hybrid Functional Differential Inclusion with Nonlocal Integral Condition}

\author{
Ahmed M. A. El-Sayed ${ }^{1,+}\left(\mathbb{D}\right.$, Wagdy G. El-Sayed ${ }^{1,+} \mathbb{D}$ and Somyya S. Amrajaa ${ }^{2, *,+}$ \\ 1 Faculty of Science, Alexandria University, Alexandria 21544, Egypt; amasayed@alexu.edu.eg (A.M.A.E.-S.); \\ wagdygoma@alexu.edu.eg (W.G.E.-S.) \\ 2 Faculty of Science, Omar Al Mukhtar University, Al Bayda 991, Libya \\ * Correspondence: Somyya44@gmail.com \\ + These authors contributed equally to this work.
}

check for updates

Citation: El-Sayed, A.M.A.; El-Sayed, W.G.; S. Amrajaa, S. On a Boundary Value Problem of Hybrid Functional Differential Inclusion with Nonlocal Integral Condition. Mathematics 2021, 9, 2667. https://doi.org/10.3390/ math9212667

Academic Editors: Changhua Lien, Hamid Reza Karimi and Sundarapandian Vaidyanathan

Received: 14 September 2021 Accepted: 17 October 2021

Published: 21 October 2021

Publisher's Note: MDPI stays neutral with regard to jurisdictional claims in published maps and institutional affiliations.

Copyright: (c) 2021 by the authors. Licensee MDPI, Basel, Switzerland. This article is an open access article distributed under the terms and conditions of the Creative Commons Attribution (CC BY) license (https:// creativecommons.org/licenses/by/ $4.0 /)$.
Abstract: In this work, we present a boundary value problem of hybrid functional differential inclusion with nonlocal condition. The boundary conditions of integral and infinite points will be deduced. The existence of solutions and its maximal and minimal will be proved. A sufficient condition for uniqueness of the solution is given. The continuous dependence of the unique solution will be studied.

Keywords: hybrid differential inclusion; boundary value problem; nonlocal condition; integral condition; infinite point boundary condition; existence of solutions; continuous dependence

MSC: 26A33; 34K45; 47G10

\section{Introduction}

Models of hybrid functional differential and integral equations have many applications (see [1-14]).

Boundary value problems with nonlocal boundary conditions have been studied by some authors (see [15-18]).

Here, we assess the boundary value problem of hybrid nonlinear functional differential inclusion with nonlocal condition.

$$
\frac{d}{d t}\left(\frac{x(t)-x(0)}{g\left(t, x\left(\phi_{1}(t)\right)\right)}\right) \in F\left(t, x\left(\phi_{2}(t)\right)\right), \quad t \in(0,1)
$$

with the nonlocal boundary condition

$$
\sum_{k=1}^{m} a_{k} x\left(\tau_{k}\right)=x_{0}, a_{k}>0 \tau_{k} \in[0,1] .
$$

The existence of solutions $x \in C[0,1]$ will be proved. The maximal and minimal solutions will be studied. A sufficient condition for uniqueness of the solution will be given. The continuous dependence of the unique solution on $x_{o}$ and on $\sum_{k=1}^{m} a_{k}$ will be proved.

Additionally, we deduce the same results for the boundary value problem of hybrid nonlinear functional differential inclusion (1) with a nonlocal integral condition

$$
\int_{0}^{1} x(s) d h(s)=x_{0}
$$

and infinite point boundary conditions

$$
\sum_{k=1}^{\infty} a_{k} x\left(\tau_{k}\right)=x_{0}, a_{k}>0 \tau_{k} \in[0,1]
$$


The following assumptions will be needed for our goals:

(I) (i) The function $\phi_{i}: I \longrightarrow I, I=[0,1]$ is continuous and $\phi_{i}(t) \leq t, i=1,2$.

(ii) The set $F: I \times R \longrightarrow 2^{R}$ is nonempty, closed, and convex for all $(t, x) \in[0,1] \times R$.

(iii) $F(t, x)$ is measurable in $t \in[0,1]$ for every $x \in R$.

(iv) $F(t, x)$ is upper semicontinuous in $x$ for every $t \in[0,1]$.

(v) There exists a bounded measurable function $a_{2}:[0,1] \longrightarrow R$ and a positive constant $K_{2}$ such that

$$
\begin{aligned}
\|F(t, x)\| & =\sup \{|f|: f \in F(t, x)\} \\
& \leq\left|a_{2}(t)\right|+K_{2}(|x|) .
\end{aligned}
$$

Remark 1. From assumptions (ii)-(iv), we can deduce that the set of selection $S_{F}$ of $F$ is nonempty (see $[1,2,5])$, that there exists $f \in F(t, x)$ such that

(vi) $f: I \times R \longrightarrow R$ is measurable in $t$ for every $x \in R$ and continuous in $x$ for $t \in[0,1]$, there exists a bounded measurable function $a_{2}:[0,1] \rightarrow R$ and a positive constant $K_{2}>0$ such that

$$
|f(t, x)| \leq\left|a_{2}(t)\right|+K_{2}(|x|),
$$

and that the function $f$ satisfies the differential equation

$$
\frac{d}{d t}\left(\frac{x(t)-x(0)}{g\left(t, x\left(\phi_{1}(t)\right)\right)}\right)=f\left(t, x\left(\phi_{2}(t)\right)\right), t \in(0,1) .
$$

Therefore, any solution of the nonlocal problem of the hybrid functional differential Equation (5) with any of the nonlocal boundary conditions (2)-(4) is a solution of the nonlocal problem of the hybrid nonlinear functional differential inclusion with any one of the nonlocal conditions (1)-(4).

(II) $\quad g: I \times R \longrightarrow R$ is measurable in $t$ for any $x \in R$ and Lipschitz in $x$ for $t \in[0,1]$, and there exists a positive constant $K_{1}>0$ such that

$$
|g(t, x)-g(t, y)| \leq K_{1}|x-y|, \forall t \in I, \text { and } x, y \in R .
$$

From assumption $(I I)$, we have

$$
|g(t, y)|-|g(t, 0)| \leq|g(t, y)-g(t, 0)| \leq K_{1}|y| .
$$

Then,

$$
\begin{aligned}
|g(t, x)| & \leq K_{1}|x|+|g(t, 0)| \\
& \leq K_{1}|x|+\mid a_{1}(t)
\end{aligned}
$$

where $\left|a_{1}(t)\right|=\sup _{t \in I}|g(t, 0)|, \quad K=\max \left\{K_{1}, K_{2}\right\}$ and

$$
|g(t, x)-g(t, 0)| \leq K_{1}|x|+|g(t, 0)| \leq K_{1}|x|+a_{1}(t) .
$$

(III) There exists a positive solution $r$ of the algebraic equation.

$$
A\left|x_{0}\right|+2(K r+a)^{2}-r=0
$$

where $A=\left(\sum_{k=1}^{m} a_{k}\right)^{-1}$. 
Definition 1. $x$ of the problem in Equations (2) and (5) is $x \in C[0,1]$ such that $\left(\frac{x(t)-x(0)}{g\left(t, x\left(\phi_{1}(t)\right)\right)}\right)$ satisfies (5).

Now, we have the following lemma.

Lemma 1. If the solution of the problems in Equations (2) and (5) exists, then it can be expressed by the integral equation

$$
\begin{aligned}
x(t) & =\frac{1}{\sum_{k=1}^{m} a_{k}}\left[x_{0}-\sum_{k=1}^{m} a_{k} g\left(\tau_{k}, x\left(\phi_{1}\left(\tau_{k}\right)\right)\right) \int_{0}^{\tau_{k}} f\left(s, x\left(\phi_{2}(s)\right)\right) d s\right] \\
& +g\left(t, x\left(\phi_{1}(t)\right)\right) \int_{0}^{t} f\left(s, x\left(\phi_{2}(s)\right)\right) d s .
\end{aligned}
$$

Proof. Let the boundary value problem in Equations (2) and (5) be satisfied; then, we can obtain

$$
x(t)=x(0)+g\left(t, x\left(\phi_{1}(t)\right)\right) \int_{0}^{t} f\left(s, x\left(\phi_{2}(s)\right)\right) d s .
$$

Putting $t=\tau$ and multiplying both sides of (7) by $a_{k}$, we obtain

$$
\sum_{k=1}^{m} a_{k} x\left(\tau_{k}\right)=\Sigma_{k=1}^{m} a_{k} x(0)+\sum_{k=1}^{m} a_{k} g\left(\tau_{k}, x\left(\phi_{1}\left(\tau_{k}\right)\right)\right) \int_{0}^{\tau_{k}} f\left(s, x\left(\phi_{2}(s)\right)\right) d s ;
$$

then

$$
x_{0}=\sum_{k=1}^{m} a_{k} x(0)+\sum_{k=1}^{m} a_{k} g\left(\tau_{k}, x\left(\left(\phi_{1}\left(\tau_{k}\right)\right)\right) \int_{0}^{\tau_{k}} f\left(s, x\left(\phi_{2}(s)\right)\right) d s\right.
$$

and

$$
x(0)=\frac{1}{\sum_{k=1}^{m} a_{k}}\left[x_{0}-\sum_{k=1}^{m} a_{k} g\left(\tau_{k}, x\left(\left(\phi_{1}\left(\tau_{k}\right)\right)\right) \int_{0}^{\tau_{k}} f\left(s, x\left(\phi_{2}(s)\right)\right) d s\right] .\right.
$$

Substituting (8) in (7), we obtain (6).

\section{Existence of Solutions}

Theorem 1. Assume that assumptions (I)-(III) are valid. Then, the integral Equation (6) has at least one solution $x \in C[0,1]$.

Proof. Define the set $Q_{r}$ by

$$
Q_{r}=\{x \in C[0,1]:\|x\| \leq r, r>0\} .
$$

Define the operator $\mathcal{F}$ by

$$
\begin{aligned}
& \mathcal{F} x(t)=\frac{1}{\sum_{k=1}^{m} a_{k}}\left[x_{0}-\sum_{k=1}^{m} a_{k} g\left(\tau_{k}, x\left(\phi_{1}\left(\tau_{k}\right)\right)\right) \int_{0}^{\tau_{k}} f\left(s, x\left(\phi_{2}(s)\right)\right) d s\right] \\
& +g\left(t, x\left(\phi_{1}(t)\right)\right) \int_{0}^{t} f\left(s, x\left(\phi_{2}(s)\right)\right) d s .
\end{aligned}
$$


Let $x \in Q_{r}$; then

$$
\begin{aligned}
& |\mathcal{F} x(t)|=\mid \frac{1}{\sum_{k=1}^{m} a_{k}}\left[x_{0}-\sum_{k=1}^{m} a_{k} g\left(\tau_{k}, x\left(\phi_{1}\left(\tau_{k}\right)\right)\right) \int_{0}^{\tau_{k}} f\left(s, x\left(\phi_{2}(s)\right)\right) d s\right] \\
& +g\left(t, x\left(\phi_{1}(t)\right)\right) \int_{0}^{t} f\left(s, x\left(\phi_{2}(s)\right)\right) d s \mid \\
& \leq \frac{\left|x_{0}\right|}{\sum_{k=1}^{m} a_{k}} \\
& +\frac{\sum_{k=1}^{m} a_{k}\left[K_{1}\left|x\left(\phi_{1}\left(\tau_{k}\right)\right)\right|+\left|a_{1}\left(\tau_{k}\right)\right|\right] \int_{0}^{\tau_{k}}\left[K_{2}\left|x\left(\phi_{2}(s)\right)\right|+\left|a_{2}(s)\right|\right] d s}{\sum_{k=1}^{m} a_{k}} \\
& +\left[K_{1}\left|x\left(\phi_{1}(t)\right)\right|+\left|a_{1}(t)\right|\right] \int_{0}^{t}\left[K_{2}\left|x\left(\phi_{2}(s)\right)\right|+\left|a_{2}(s)\right|\right] d s \\
& \leq \frac{\left|x_{0}\right|}{\sum_{k=1}^{m} a_{k}}+[K\|x\|+a][K\|x\|+a] \int_{0}^{1} d s+[K\|x\|+a][K\|x\|+a] \int_{0}^{1} d s \\
& \leq \frac{\left|x_{0}\right|}{\sum_{k=1}^{m} a_{k}}+2[K r+a][K r+a] \\
& \leq A\left|x_{0}\right|+2(K r+a)^{2}=r .
\end{aligned}
$$

Thus, the class of functions $\{\mathcal{F} x\}$ is uniformly bounded on $Q_{r}$ and $\mathcal{F}: Q_{r} \rightarrow Q_{r}$. Let $x \in Q_{r}$ and $t_{1}, t_{2} \in[0,1]$ such that $\left|t_{2}-t_{1}\right|<\delta$; then,

$$
\begin{aligned}
& \left|\mathcal{F} x\left(t_{2}\right)-\mathcal{F} x\left(t_{1}\right)\right|=\mid \frac{1}{\sum_{k=1}^{m} a_{k}}\left[x_{0}-\sum_{k=1}^{m} a_{k} g\left(\tau_{k}, x\left(\phi_{1}\left(\tau_{k}\right)\right)\right) \int_{0}^{\tau_{k}} f\left(s, x\left(\phi_{2}(s)\right)\right) d s\right] \\
& +g\left(t_{2}, x\left(\phi_{1}\left(t_{2}\right)\right)\right) \int_{0}^{t_{2}} f\left(s, x\left(\phi_{2}(s)\right)\right) d s \\
& -\left(\frac{1}{\sum_{k=1}^{m} a_{k}}\left[x_{0}-\sum_{k=1}^{m} a_{k} g\left(\tau_{k}, x\left(\phi_{1}\left(\tau_{k}\right)\right)\right) \int_{0}^{\tau_{k}} f\left(s, x\left(\phi_{2}(s)\right)\right) d s\right]\right. \\
& \left.+g\left(t_{1}, x\left(\phi_{1}\left(t_{1}\right)\right)\right) \int_{0}^{t_{1}} f\left(s, x\left(\phi_{2}(s)\right)\right) d s\right) \mid \\
& \leq\left|g\left(t_{2}, x\left(\phi_{1}\left(t_{2}\right)\right)\right) \int_{0}^{t_{2}} f\left(s, x\left(\phi_{2}(s)\right)\right) d s-g\left(t_{1}, x\left(\phi_{1}\left(t_{1}\right)\right)\right) \int_{0}^{t_{1}} f\left(s, x\left(\phi_{2}(s)\right)\right) d s\right| \\
& \leq \mid g\left(t_{2}, x\left(\phi_{1}\left(t_{2}\right)\right)\right) \int_{0}^{t_{2}} f\left(s, x\left(\phi_{2}(s)\right)\right) d s-g\left(t_{2}, x\left(\phi_{1}\left(t_{1}\right)\right)\right) \int_{0}^{t_{1}} f\left(s, x\left(\phi_{2}(s)\right)\right) d s \\
& +g\left(t_{2}, x\left(\phi_{1}\left(t_{1}\right)\right)\right) \int_{0}^{t_{1}} f\left(s, x\left(\phi_{2}(s)\right)\right) d s-g\left(t_{1}, x\left(\phi_{1}\left(t_{1}\right)\right)\right) \int_{0}^{t_{1}} f\left(s, x\left(\phi_{2}(s)\right)\right) d s \mid \\
& \leq\left|g\left(t_{2}, x\left(\phi_{1}\left(t_{2}\right)\right)\right)-g\left(t_{2}, x\left(\phi_{1}\left(t_{1}\right)\right)\right)\right| \int_{t_{1}}^{t_{2}}\left|f\left(s, x\left(\phi_{2}(s)\right)\right)\right| d s \\
& +\left|g\left(t_{2}, x\left(\phi_{1}\left(t_{1}\right)\right)\right)-g\left(t_{1}, x\left(\phi_{1}\left(t_{1}\right)\right)\right)\right| \int_{0}^{t_{1}}\left|f\left(s, x\left(\phi_{2}(s)\right)\right)\right| d s \\
& \leq K_{1}\left|x\left(\phi_{1}\left(t_{2}\right)\right)-x\left(\phi_{1}\left(t_{1}\right)\right)\right| \int_{t_{1}}^{t_{2}}\left(\left|a_{2}(s)\right|+K_{2}\left|x\left(\phi_{2}(s)\right)\right|\right) d s \\
& +\left|g\left(t_{2}, x\left(\phi_{1}\left(t_{1}\right)\right)\right)-g\left(t_{1}, x\left(\phi_{1}\left(t_{1}\right)\right)\right)\right| \int_{0}^{t_{1}}\left(\left|a_{2}(s)\right|+K_{2}\left|x\left(\phi_{2}(s)\right)\right|\right) d s \\
& \leq K\left|x\left(\phi_{1}\left(t_{1}\right)\right)-x\left(\phi_{1}\left(t_{2}\right)\right)\right| \int_{t_{1}}^{t_{2}}\left(a+K\left|x\left(\phi_{2}(s)\right)\right|\right) d s \\
& +\left|g\left(t_{2}, x\left(\phi_{1}\left(t_{1}\right)\right)\right)-g\left(t_{1}, x\left(\phi_{1}\left(t_{1}\right)\right)\right)\right| \int_{0}^{t_{1}}\left(a+K\left|x\left(\phi_{2}(s)\right)\right|\right) d s .
\end{aligned}
$$

Thus, the class of functions $\{\mathcal{F} x\}$ is equicontinuous on $Q_{r}$ and $\{\mathcal{F} y\}$ is a compact operator by the Arzela-Ascoli Theorem [19]. 
Now, we prove that $\mathcal{F}$ is a continuous operator. Let $x_{n} \subset Q_{r}$ be a convergent sequence such that $x_{n} \rightarrow x$; then,

$$
\begin{aligned}
& \mathcal{F} x_{n}(t)=\frac{1}{\sum_{k=1}^{m} a_{k}}\left[x_{0}-\sum_{k=1}^{m} a_{k} g\left(\tau_{k}, x_{n}\left(\phi_{1}\left(\tau_{k}\right)\right)\right) \int_{0}^{\tau_{k}} f\left(s, x_{n}\left(\phi_{2}(s)\right)\right) d s\right] \\
& +g\left(t, x_{n}\left(\phi_{1}(t)\right)\right) \int_{0}^{t} f\left(s, x_{n}\left(\phi_{2}(s)\right)\right) d s .
\end{aligned}
$$

Using Lebesgue-dominated convergence Theorem [19] and assumptions (iv)-(III), we have

$$
\begin{aligned}
& \lim _{n \rightarrow \infty} \mathcal{F} x_{n}(t)=\lim _{n \rightarrow \infty}\left(\frac{1}{\sum_{k=1}^{m} a_{k}}\left[x_{0}-\sum_{k=1}^{m} a_{k} g\left(\tau_{k}, x_{n}\left(\phi_{1}\left(\tau_{k}\right)\right)\right) \int_{0}^{\tau_{k}} f\left(s, x_{n}\left(\phi_{2}(s)\right)\right) d s\right]\right. \\
& \left.+g\left(t, x_{n}\left(\phi_{1}(t)\right)\right) \int_{0}^{t} f\left(s, x_{n}\left(\phi_{2}(s)\right)\right) d s\right) \\
& =\frac{1}{\sum_{k=1}^{m} a_{k}}\left[x_{0}-\sum_{k=1}^{m} a_{k} g\left(\tau_{k}, \lim _{n \rightarrow \infty} x_{n}\left(\phi_{1}\left(\tau_{k}\right)\right)\right) \int_{0}^{\tau_{k}} f\left(s, \lim _{n \rightarrow \infty} x_{n}\left(\phi_{2}(s)\right)\right) d s\right] \\
& +g\left(t, \lim _{n \rightarrow \infty} x_{n}\left(\phi_{1}(t)\right)\right) \int_{0}^{t} f\left(s, \lim _{n \rightarrow \infty} x_{n}\left(\phi_{2}(s)\right)\right) d s . \\
& =\frac{1}{\sum_{k=1}^{m} a_{k}}\left[x_{0}-\sum_{k=1}^{m} a_{k} g\left(\tau_{k}, x\left(\phi_{1}\left(\tau_{k}\right)\right)\right) \int_{0}^{\tau_{k}} f\left(s, x\left(\phi_{2}(s)\right)\right) d s\right] \\
& +g\left(t, x\left(\phi_{1}(t)\right)\right) \int_{0}^{t} f\left(s, x\left(\phi_{2}(s)\right)\right) d s \\
& =\mathcal{F} x(t) .
\end{aligned}
$$

Then, $\mathcal{F}: Q_{r} \rightarrow Q_{r}$ is continuous, and by Schauder fixed point Theorem [19], there exists at least one solution $x \in C[0,1]$ of $(6)$.

Now,

$$
\begin{aligned}
& x(t)=x(0)+g\left(t, x\left(\phi_{1}(t)\right)\right) \int_{0}^{t} f\left(s, x\left(\phi_{2}(s)\right)\right) d s \\
& \frac{x(t)-x(0)}{g\left(t, x\left(\phi_{1}(t)\right)\right)}=\int_{0}^{t} f\left(s, x\left(\phi_{2}(s)\right)\right) d s \\
& \frac{d}{d t}\left(\frac{x(t)-x(0)}{g\left(t, x\left(\phi_{1}(t)\right)\right)}\right)=\frac{d}{d t} \int_{0}^{t} f\left(s, x\left(\phi_{2}(s)\right)\right) d s \\
& \frac{d}{d t}\left(\frac{x(t)-x(0)}{g\left(t, x\left(\phi_{1}(t)\right)\right)}\right)=f\left(t, x\left(\phi_{2}(t)\right)\right) .
\end{aligned}
$$

putting $t=\tau_{k}$ and multiplying by $\sum_{k=1}^{m} a_{k}$ in (6), we obtain

$$
\begin{aligned}
& \sum_{k=1}^{m} a_{k} x\left(\tau_{k}\right)=\sum_{k=1}^{m} a_{k}\left(\frac{1}{\sum_{k=1}^{m} a_{k}}\left[x_{0}-\sum_{k=1}^{m} a_{k} g\left(\tau_{k}, x\left(\phi_{1}\left(\tau_{k}\right)\right)\right) \int_{0}^{\tau_{k}} f\left(s, x\left(\phi_{2}(s)\right)\right) d s\right]\right. \\
& \left.+g\left(\tau_{k}, x\left(\phi_{1}\left(\tau_{k}\right)\right)\right) \int_{0}^{\tau_{k}} f\left(s, x\left(\phi_{2}(s)\right)\right) d s\right) .
\end{aligned}
$$

Then,

$$
\sum_{k=1}^{m} a_{k} x\left(\tau_{k}\right)=x_{0}, a_{k}>0, \tau_{k} \in[0,1]
$$

This proves the equivalence between the problem in Equations (2) and (5) and the integral Equation (6). Then, there exists at least one solution $x \in C[0,1]$ of the hybrid nonlinear functional differential Equation (5) with the nonlocal condition (2). Consequently, there 
exists at least one solution $x \in C[0,1]$ of the nonlocal problem of the hybrid nonlinear functional differential inclusion (1) with the nonlocal condition (2).

\section{Maximal and Minimal Solutions}

Here, we study the maximal and minimal solutions for the problem in Equations (2) and (5). Let $u(t)$ be a solution of (6); then, $u(t)$ is said to be a maximal solution of (6) if it satisfies the inequality

$$
x(t) \leq u(t), \quad t \in[0,1] .
$$

A minimal solution $v(t)$ can be defined in a similar way by reversing the above inequality i.e.,

$$
x(t)>v(t), \quad t \in[0,1] .
$$

Lemma 2. Let the assumptions of Theorem 1 be satisfied. Assume that $x$ and $y$ are two continuous functions on $[0,1]$ satisfying.

$$
\begin{aligned}
& x(t)<\frac{1}{\sum_{k=1}^{m} a_{k}}\left[x_{0}-\sum_{k=1}^{m} a_{k} g\left(\tau_{k}, x\left(\phi_{1}\left(\tau_{k}\right)\right)\right) \int_{0}^{\tau_{k}} f\left(s, x\left(\phi_{2}(s)\right)\right) d s\right] \\
& +g\left(t, x\left(\phi_{1}(t)\right)\right) \int_{0}^{t} f\left(s, x\left(\phi_{2}(s)\right)\right) d s \quad t \in[0,1] . \\
& y(t)>\frac{1}{\sum_{k=1}^{m} a_{k}}\left[x_{0}-\sum_{k=1}^{m} a_{k} g\left(\tau_{k}, y\left(\phi_{1}\left(\tau_{k}\right)\right)\right) \int_{0}^{\tau_{k}} f\left(s, y\left(\phi_{2}(s)\right)\right) d s\right] \\
& +g\left(t, y\left(\phi_{1}(t)\right)\right) \int_{0}^{t} f\left(s, y\left(\phi_{2}(s)\right)\right) d s t \in[0,1]
\end{aligned}
$$

where one of them is strict.

Let the functions $f$ and $g$ be monotonically nondecreasing in $x$; then,

$$
x(t)<y(t), \quad t>0 .
$$

Proof. Let the conclusion (9) be untrue; then, there exists $t_{1}$ with

$$
x\left(t_{1}\right)<y\left(t_{1}\right), \quad t_{1}>0 \quad \text { and } x(t)<y(t), \quad 0<t<t_{1} .
$$

If $f$ and $g$ are monotonic functions in $x$, we have

$$
\begin{aligned}
& x\left(t_{1}\right) \leq \frac{1}{\sum_{k=1}^{m} a_{k}}\left[x_{0}-\sum_{k=1}^{m} a_{k} g\left(\tau_{k}, x\left(\phi_{1}\left(\tau_{k}\right)\right)\right) \int_{0}^{\tau_{k}} f\left(s, x\left(\phi_{2}(s)\right)\right) d s\right] \\
& +g\left(t_{1}, x\left(\phi_{1}\left(t_{1}\right)\right)\right) \int_{0}^{t_{1}} f\left(s, x\left(\phi_{2}(s)\right)\right) d s \quad t_{1} \in[0,1] . \\
& \geq \frac{1}{\sum_{k=1}^{m} a_{k}}\left[x_{0}-\sum_{k=1}^{m} a_{k} g\left(\tau_{k}, y\left(\phi_{1}\left(\tau_{k}\right)\right)\right) \int_{0}^{\tau_{k}} f\left(s, y\left(\phi_{2}(s)\right)\right) d s\right] \\
& +g\left(t_{1}, y\left(\phi_{1}\left(t_{1}\right)\right)\right) \int_{0}^{t_{1}} f\left(s, y\left(\phi_{2}(s)\right)\right) d s \quad t_{1} \in[0,1]<y\left(t_{1}\right) .
\end{aligned}
$$

This contradicts the fact that $x\left(t_{1}\right)=y\left(t_{1}\right)$. This completes the proof.

For the continuous maximal and minimal solutions for (6), we have the following theorem.

Theorem 2. Let the assumptions of Theorem 1 hold. Moreover, if $f$ and $g$ are monotonically nondecreasing functions in $x$ for each $t \in[0,1]$, then Equation (6) has maximal and minimal solutions. 
Proof. First, we must demonstrate the existence of the maximal solution of (6). Let $\epsilon>0$ be given. Now, consider the integral equation

$$
\begin{aligned}
x_{\epsilon}(t) \leq & \frac{1}{\sum_{k=1}^{m} a_{k}}\left[x_{0}-\sum_{k=1}^{m} a_{k} g\left(\tau_{k}, x_{\epsilon}\left(\phi_{1}\left(\tau_{k}\right)\right)\right) \int_{0}^{\tau_{k}} f\left(s, x_{\epsilon}\left(\phi_{2}(s)\right)\right) d s\right] \\
& +g\left(t, x_{\epsilon}\left(\phi_{1}(t)\right)\right) \int_{0}^{t} f\left(s, x_{\epsilon}\left(\phi_{2}(s)\right)\right) d s \quad t \in[0,1]
\end{aligned}
$$

where

$$
\begin{aligned}
& f\left(t, x_{\epsilon}\left(\phi_{2}(t)\right)\right)=f\left(s, x_{\epsilon}\left(\phi_{2}(t)\right)\right)+\epsilon, \\
& g\left(s, x_{\epsilon}\left(\phi_{1}(t)\right)\right)=f\left(s, x_{\epsilon}\left(\phi_{1}(t)\right)\right)+\epsilon .
\end{aligned}
$$

Let $\epsilon_{1}, \epsilon_{2}$ be such that $0<\epsilon_{2}<\epsilon_{1}$; then,

$$
\begin{gathered}
x_{\epsilon_{2}}(t)=\frac{1}{\sum_{k=1}^{m} a_{k}}\left[x_{0}-\sum_{k=1}^{m} a_{k} g\left(\tau_{k}, x_{\epsilon_{1}}\left(\phi_{1}\left(\tau_{k}\right)\right)\right) \int_{0}^{\tau_{k}} f\left(s, x_{\epsilon_{2}}\left(\phi_{2}(s)\right)\right) d s\right] \\
+g\left(t, x_{\epsilon_{2}}\left(\phi_{1}(t)\right)\right) \int_{0}^{t} f\left(s, x_{\epsilon_{2}}\left(\phi_{2}(s)\right)\right) d s \quad t \in[0,1] . \\
x_{\epsilon_{2}}(t)=\frac{1}{\sum_{k=1}^{m} a_{k}}\left[x_{0}-\sum_{k=1}^{m} a_{k} g\left(\tau_{k}, x_{\epsilon_{2}}\left(\phi_{1}\left(\tau_{k}\right)\right)+\epsilon_{2}\right) \int_{0}^{\tau_{k}}\left(f\left(s, x_{\epsilon_{2}}\left(\phi_{2}(s)\right)\right)+\epsilon_{2}\right) d s\right] \\
+g\left(t, x_{\epsilon_{2}}\left(\phi_{1}(t)\right)+\epsilon_{2}\right) \int_{0}^{t}\left(f\left(s, x_{\epsilon_{2}}\left(\phi_{2}(s)\right)\right)+\epsilon_{2}\right) d s \quad t \in[0,1] .
\end{gathered}
$$

Additionally,

$$
\begin{aligned}
& x_{\epsilon_{1}}(t)=\frac{1}{\sum_{k=1}^{m} a_{k}}\left[x_{0}-\sum_{k=1}^{m} a_{k} g\left(\tau_{k}, x_{\epsilon_{1}}\left(\phi\left(\tau_{k}\right)\right)\right) \int_{0}^{\tau_{k}} f\left(s, x_{\epsilon_{1}}\left(\phi_{2}(s)\right)\right) d s\right] \\
& +g\left(t, x_{\epsilon_{1}}\left(\phi_{1}(t)\right)\right) \int_{0}^{t} f\left(s, x_{\epsilon_{1}}\left(\phi_{2}(s)\right)\right) d s \quad t \in[0,1] . \\
& x_{\epsilon_{1}}(t)=\frac{1}{\sum_{k=1}^{m} a_{k}}\left[x_{0}-\sum_{k=1}^{m} a_{k}\left(g\left(\tau_{k}, x_{\epsilon_{1}}\left(\phi_{1}\left(\tau_{k}\right)\right)+\epsilon_{1}\right) \int_{0}^{\tau_{k}}\left(f\left(s, x_{\epsilon_{1}}\left(\phi_{2}(s)\right)\right)+\epsilon_{1}\right) d s\right]\right. \\
& \left.+\left(g\left(t, x_{\epsilon_{1}}\left(\phi_{1}(t)\right)+\epsilon_{1}\right) \int_{0}^{t} f\left(s, x_{\epsilon_{1}}\left(\phi_{2}(s)\right)\right)+\epsilon_{1}\right)\right) d s t \in[0,1] .
\end{aligned}
$$

Then,

$$
\begin{aligned}
& x_{\epsilon_{1}}>\frac{1}{\sum_{k=1}^{m} a_{k}}\left[x_{0}-\sum_{k=1}^{m} a_{k} g\left(\tau_{k}, x_{\epsilon_{2}}\left(\phi_{1}\left(\tau_{k}\right)\right)\right) \int_{0}^{\tau_{k}}\left(f\left(s, x_{\epsilon_{2}}\left(\phi_{2}(s)\right)\right)\right) d s\right] \\
& +g\left(t, x_{\epsilon_{2}}\left(\phi_{1}(t)\right)\right) \int_{0}^{t}\left(f\left(s, x_{\epsilon_{2}}\left(\phi_{2}(s)\right)\right)\right) d s \quad t \in[0,1] .
\end{aligned}
$$

Applying Lemma 2, we obtain

$$
x_{\epsilon_{2}}<x_{\epsilon_{1}}, t \in[0,1] .
$$

As shown before, the family of function $x_{\epsilon}(t)$ is equi-continuous and uniformly bounded; then, by the Arzela Theorem, there exists a decreasing sequence $\epsilon_{n}$ such that $\epsilon_{0} \rightarrow 0$ as $n \rightarrow \infty$, and $u(t)=\lim _{n \rightarrow \infty} x_{\epsilon_{n}}(t)$ exists uniformly in $[0,1]$, and denote this limit by $u(t)$. From the continuity of the functions, $f_{\epsilon}\left(t, x_{\epsilon}\left(\phi_{2}(t)\right)\right)$, we get

$$
\begin{aligned}
& f_{\epsilon}\left(t, x_{\epsilon}\left(\phi_{2}(t)\right)\right) \longrightarrow f\left(t, x\left(\phi_{2}(t)\right)\right) \text { as } n \rightarrow \infty, \\
& g_{\epsilon}\left(t, x_{\epsilon}\left(\phi_{2}(t)\right)\right) \longrightarrow g\left(t, x\left(\phi_{2}(t)\right)\right) \text { as } n \rightarrow \infty
\end{aligned}
$$


and

$$
\begin{aligned}
& \left.u(t)=\lim _{n \rightarrow \infty} x_{\epsilon_{n}}(t)=\frac{1}{\sum_{k=1}^{m} a_{k}}\left[x_{0}-\sum_{k=1}^{m} a_{k} g\left(\tau_{k}, u\left(\phi_{1}\left(\tau_{k}\right)\right)\right) \int_{0}^{\tau_{k}}\left(f\left(s, u\left(\phi_{2}(s)\right)\right)\right)\right) d s\right] \\
& +g\left(t, u\left(\phi_{1}(t)\right)\right) \int_{0}^{t}\left(f\left(s, u\left(\phi_{2}(s)\right)\right)\right) d s \quad t \in[0,1] .
\end{aligned}
$$

Now, we prove that $u(t)$ is the maximal solution of (6). To do this, let $x(t)$ be any solution of (6); then,

$$
\begin{aligned}
& x(t)=\frac{1}{\sum_{k=1}^{m} a_{k}}\left[x_{0}-\sum_{k=1}^{m} a_{k} g\left(\tau_{k}, x\left(\phi_{1}\left(\tau_{k}\right)\right)\right) \int_{0}^{\tau_{k}} f\left(s, x\left(\phi_{2}(s)\right)\right) d s\right] \\
& +g\left(t, x\left(\phi_{1}(t)\right)\right) \int_{0}^{t} f\left(s, x\left(\phi_{2}(s)\right)\right) d s \quad t \in[0,1]
\end{aligned}
$$

and

$$
\begin{aligned}
& x_{\epsilon}(t)=\frac{1}{\sum_{k=1}^{m} a_{k}}\left[x_{0}-\sum_{k=1}^{m} a_{k} g\left(\tau_{k}, x_{\epsilon}\left(\phi_{1}\left(\tau_{k}\right)\right)\right) \int_{0}^{\tau_{k}} f\left(s, x_{\epsilon}\left(\phi_{2}(s)\right)\right) d s\right] \\
& +g\left(t, x_{\epsilon}\left(\phi_{1}(t)\right)\right) \int_{0}^{t} f\left(s, x_{\epsilon}\left(\phi_{2}((s)) d s \quad t \in[0,1] .\right.\right. \\
& =\frac{1}{\sum_{k=1}^{m} a_{k}}\left[x_{0}-\sum_{k=1}^{m} a_{k} g\left(\tau_{k}, x_{\epsilon}\left(\phi_{1}\left(\tau_{k}\right)\right)+\epsilon\right) \int_{0}^{\tau_{k}}\left(f\left(s, x_{\epsilon}\left(\phi_{2}(s)\right)+\epsilon\right) d s\right]\right. \\
& +g\left(t, x_{\epsilon}\left(\phi_{1}(t)\right)+\epsilon\right) \int_{0}^{t} f\left(s, x_{\epsilon}\left(\phi_{2}(s)\right)+\epsilon\right) d s \quad t \in[0,1] .
\end{aligned}
$$

Then,

$$
\begin{aligned}
& x_{\epsilon}(t)>\frac{1}{\sum_{k=1}^{m} a_{k}}\left[x_{0}-\sum_{k=1}^{m} a_{k} g\left(\tau_{k}, x_{\epsilon}\left(\phi_{1}\left(\tau_{k}\right)\right)\right) \int_{0}^{\tau_{k}} f\left(s, x_{\epsilon}\left(\phi_{2}(s)\right)\right) d s\right] \\
& +g\left(t, x_{\epsilon}\left(\phi_{1}(t)\right)\right) \int_{0}^{t} f\left(s, x_{\epsilon}\left(\phi_{2}(s)\right)\right) d s \quad t \in[0,1] .
\end{aligned}
$$

Applying Lemma 2, we obtain

$$
x(t)<x_{\epsilon}(t), t \in[0,1] .
$$

From the uniqueness of the maximal solution, it clear that $x_{\epsilon}(t)$ tends to $u(t)$ uniformly in $[0,1]$ as $\epsilon \rightarrow 0$ in a similar way as above and we can prove the existence of the minimal solution.

\section{Uniqueness of the Solution}

Here, we study a sufficient condition for the uniqueness of the solution $x \in C[0,1]$ of the problem in Equations (2) and (5).

Consider the following assumptions:

(I*) (i) The function $\phi_{i}: I \longrightarrow I$ is continuous, and $\phi_{i}(t) \leq t, \quad i=1,2$.

(ii) The set $F(t, x)$ is nonempty, compact, and convex for all $(t, x) \in[0,1] \times R$.

(iii) $F(t, x)$ is measurable in $t \in[0,1]$ for every $x \in R$ and satisfies the Lipschitz condition with a positive constant $K_{2}$ such that

$$
H\left(F(t, x), F(t, y) \mid \leq K_{2}(|x-y|)\right.
$$

where $H(A, B)$ is the Hausdorff metric between the two subsets $A, B \in I \times E$ (see [16]). 
Remark 2. From this assumptions, we can deduce that there exists a function $f \in F(t, x)$ such that

(iv) $f: I \times R \rightarrow R$ is measurable in $t \in[0,1]$ for every $x \in R$ and satisfies the Lipschitz condition with a positive constant $K_{1}$ such that (see [19-21])

$$
|f(t, x)-f(t, y)| \leq K_{2}(|x-y|) \text {. }
$$

$\left(I I^{*}\right) g: I \times R \longrightarrow R$ is continuous and satisfies the Lipschitz condition with positive constant $K_{1}$ such that

$$
|g(t, x)-g(t, y)| \leq K_{1}|x-y| .
$$

From the assumption $\left(I^{*}\right)$, we have

$$
|f(t, x)|-|f(t, 0)| \leq|f(t, x)-f(t, 0)| \leq K_{2}(|x|) .
$$

Then,

$$
\begin{aligned}
|f(t, x)| & \leq K_{1}(|x|)+|f(t, 0)| \\
& \leq K_{2}(|x|)+\mid a_{2}(t)
\end{aligned}
$$

where $\left|a_{2}(t)\right|=\sup _{t \in I}|f(t, 0)|$.

From assumption $\left(I I^{*}\right)$, we have

$$
|g(t, y)|-|g(t, 0)| \leq|g(t, y)-g(t, 0)| \leq K_{1}|y|
$$

Then,

$$
\begin{aligned}
|g(t, x)| & \leq K_{1}|x|+|g(t, 0)| \\
& \leq K_{1}|x|+\mid a_{1}(t)
\end{aligned}
$$

where $\left|a_{1}(t)\right|=\sup _{t \in I}|g(t, 0)|, \quad K=\max \left\{K_{1}, K_{2}\right\}$

Theorem 3. Let the assumptions $\left(I^{*}\right)-\left(I I^{*}\right)$ be satisfied. If $\left(1-4 K^{2} r-4 a K\right) \leq 1$, then the solution of the problem in Equations (2) and (5) is unique.

Proof. Let $x_{1}$ and $x_{2}$ be two solutions of the problem in Equations (2) and (5); then, 


$$
\begin{aligned}
& \left.\mid x_{1}(t)-x_{2}(t)\right)|=| \frac{1}{\sum_{k=1}^{m} a_{k}}\left[x_{0}-\sum_{k=1}^{m} a_{k} g\left(\tau_{k}, x_{1}\left(\phi_{1}\left(\tau_{k}\right)\right)\right) \int_{0}^{\tau_{k}} f\left(s, x_{1}\left(\phi_{2}(s)\right)\right) d s\right] \\
& \quad+g\left(t, x_{1}\left(\phi_{1}(t)\right)\right) \int_{0}^{t} f\left(s, x_{1}\left(\phi_{2}((s)) d s\right.\right. \\
& \quad-\left(\frac{1}{\sum_{k=1}^{m} a_{k}}\left[x_{0}-\sum_{k=1}^{m} a_{k} g\left(\tau_{k}, x_{2}\left(\phi_{1}\left(\tau_{k}\right)\right)\right) \int_{0}^{\tau_{k}} f\left(s, x_{2}\left(\phi_{2}(s)\right)\right) d s\right]\right. \\
& \left.\quad+g\left(t, x_{2}\left(\phi_{1}(t)\right)\right) \int_{0}^{t} f\left(s, x_{2}\left(\phi_{2}(s)\right)\right) d s\right) \mid \\
& \leq \frac{\left|\sum_{k=1}^{m} a_{k}\left(g\left(\tau_{k}, x_{1}\left(\phi_{1}\left(\tau_{k}\right)\right)\right) \int_{0}^{\tau_{k}} f\left(s, x_{1}\left(\phi_{2}(s)\right)\right) d s-g\left(\tau_{k}\right), x_{2}\left(\phi_{1}\left(\tau_{k}\right)\right)\right) \int_{0}^{\tau_{k}} f\left(s, x_{2}\left(\phi_{2}(s)\right)\right) d s\right|}{\sum_{k=1}^{m} a_{k}} \\
& +g\left(t, x_{1}\left(\phi_{1}(t)\right) \int_{0}^{t} f\left(s, x_{1}\left(\phi_{2}(s)\right)\right) d s-g\left(\tau_{k}, x_{2}\left(\phi_{1}(t)\right)\right) \int_{0}^{t} f\left(s, x_{2}\left(\phi_{2}(s)\right)\right) d s \mid\right. \\
& \leq \frac{\left|\sum_{k=1}^{m} a_{k}\left(g\left(\tau_{k}, x_{1}\left(\phi_{1}\left(\tau_{k}\right)\right)\right) \int_{0}^{\tau_{k}} f\left(s, x_{1}\left(\phi_{2}(s)\right)\right) d s-g\left(\tau_{k}\right), x_{2}\left(\phi_{1}\left(\tau_{k}\right)\right)\right) \int_{0}^{\tau_{k}} f\left(s, x_{1}\left(\phi_{2}(s)\right)\right) d s\right|}{\sum_{k=1}^{m} a_{k}} \\
& \leq \frac{\mid \sum_{k=1}^{m} a_{k}\left(g\left(\tau_{k}, x_{2}\left(\phi_{1}\left(\tau_{k}\right)\right)\right) \int_{0}^{\tau_{k}} f\left(s, x_{1}\left(\phi_{2}(s)\right)\right) d s-g\left(\tau_{k}, x_{2}\left(\phi_{1}\left(\tau_{k}\right)\right)\right) \int_{0}^{\tau_{k}} f\left(s, x_{2}\left(\phi_{2}(s)\right)\right) d s \mid\right.}{\sum_{k=1}^{m} a_{k}} \\
& \quad+\left|g\left(t, x_{1}\left(\phi_{1}(t)\right)\right) \int_{0}^{t} f\left(s, x_{1}\left(\phi_{2}(s)\right)\right) d s-g\left(t, x_{2}\left(\phi_{1}(t)\right)\right) \int_{0}^{t} f\left(s, x_{1}\left(\phi_{2}(s)\right)\right) d s\right| \\
& \quad+\left|g\left(t, x_{2}\left(\phi_{1}(t)\right)\right) \int_{0}^{t} f\left(s, x_{1}\left(\phi_{2}(s)\right)\right) d s-g\left(t, x_{2}\left(\phi_{1}(t)\right)\right) \int_{0}^{t} f\left(s, x_{2}\left(\phi_{2}(s)\right)\right) d s\right| \\
& \leq \frac{\sum_{k=1}^{m} a_{k} K\left\|x_{1}-x_{2}\right\|\left(K\left\|x_{1}\right\|+a\right)}{\sum_{k=1}^{m} a_{k}} \\
& \quad+\frac{\sum_{k=1}^{m} a_{k} K\left\|x_{1}-x_{2}\right\|\left(K\left\|x_{2}\right\|+a\right)}{\sum_{k=1}^{m} a_{k}} \\
& +K\left\|x_{1}-x_{2}\right\|\left(K\left\|x_{1}\right\|+a\right)+K\left\|x_{1}-x_{2}\right\|\left(K\left\|x_{2}\right\|+a\right) \\
& \leq \\
& \leq \\
& \leq
\end{aligned}
$$

Then,

$$
\left\|x_{1}-x_{2}\right\|\left(1-4 K^{2} r-4 a K\right) \leq 0,
$$

Since $\left(1-4 K^{2} r-4 a K\right) \leq 1, x_{1}(t)=x_{2}(t)$ and the solution of (5) and (2) is unique.

\section{Continuous Dependence of the Solution}

Definition 2. The unique solution of the problem in Equations (2) and (5) depends continuously on initial data $x_{0}$, if $\epsilon>0, \exists \delta>0$, such that

$$
\left|x_{0}-x_{0}^{*}\right| \leq \delta \quad \Rightarrow\left\|x-x^{*}\right\| \leq \epsilon
$$

where $x^{*}$ is the unique solution of the integral equation

$$
\begin{aligned}
& x^{*}(t)=\frac{1}{\sum_{k=1}^{m} a_{k}}\left[x_{0}^{*}-\sum_{k=1}^{m} a_{k} g\left(\tau_{k}, x^{*}\left(\phi_{1}\left(\tau_{k}\right)\right)\right) \int_{0}^{\tau_{k}} f\left(s, x^{*}\left(\phi_{2}(s)\right)\right) d s\right] \\
& +g\left(t, x^{*}\left(\phi_{1}(t)\right) \int_{0}^{t} f\left(s, x^{*}\left(\phi_{2}(s)\right) d s .\right.\right.
\end{aligned}
$$

Theorem 4. Let the assumptions $\left(I^{*}\right)-\left(I I^{*}\right)$ be satisfied; then, the unique solution of (5) and (2) depends continuously on $x_{0}$ 
Proof. Let $x$ and $x^{*}$ be the solutions of the problem in Equations (2) and (5); then,

$$
\begin{aligned}
& \left.\left|x(t)-x^{*}(t)\right|=\mid \frac{1}{\sum_{k=1}^{m} a_{k}}\left[x_{0}-\sum_{k=1}^{m} a_{k} g\left(\tau_{k}, x\left(\phi_{1}\left(\tau_{k}\right)\right)\right) \int_{0}^{\tau_{k}} f\left(s, x\left(\phi_{2}(s)\right)\right)\right) d s\right] \\
& \left.+g\left(t, x\left(\phi_{1}(t)\right)\right) \int_{0}^{t} f\left(s, x\left(\phi_{2}(s)\right)\right)\right) d s \\
& \left.-\frac{1}{\sum_{k=1}^{m} a_{k}}\left[x_{0}^{*}-\sum_{k=1}^{m} a_{k} g\left(\tau_{k}, x^{*}\left(\phi_{1}\left(\tau_{k}\right)\right)\right) \int_{0}^{\tau_{k}} f\left(s, x^{*}\left(\phi_{2}(s)\right)\right)\right) d s\right] \\
& \left.\left.-g\left(t, x^{*}\left(\phi_{1}(t)\right)\right) \int_{0}^{t} f\left(s, x^{*}\left(\phi_{2}(s)\right)\right)\right) d s\right) \mid \\
& \leq \frac{\left|x_{0}-x_{0}^{*}\right|}{\sum_{k=1}^{m} a_{k}} \\
& \leq \frac{\left|\sum_{k=1}^{m} a_{k}\left(g\left(\tau_{k}, x\left(\phi_{1}\left(\tau_{k}\right)\right)\right) \int_{0}^{\tau_{k}} f\left(s,\left(\phi_{2} x(s)\right)\right) d s-g\left(\tau_{k}\right), x^{*}\left(\phi_{1}\left(\tau_{k}\right)\right)\right) \int_{0}^{\tau_{k}} f\left(s, x^{*}\left(\phi_{2}(s)\right)\right) d s\right|}{\sum_{k=1}^{m} a_{k}} \\
& +g\left(t, x\left(\phi_{1}(t)\right) \int_{0}^{t} f\left(s, x\left(\phi_{2}(s)\right)\right) d s-g\left(\tau_{k}, x^{*}\left(\phi_{1}(t)\right)\right) \int_{0}^{t} f\left(s, x^{*}\left(\phi_{2}(s)\right)\right) d s \mid\right. \\
& \leq \delta A \\
& +\frac{\left|\sum_{k=1}^{m} a_{k}\left(g\left(\tau_{k}, x\left(\phi_{1}\left(\tau_{k}\right)\right)\right) \int_{0}^{\tau_{k}} f\left(s, x\left(\phi_{2}(s)\right)\right) d s-g\left(\tau_{k}\right), x^{*}\left(\phi_{1}\left(\tau_{k}\right)\right)\right) \int_{0}^{\tau_{k}} f\left(s, x\left(\phi_{2}(s)\right)\right) d s\right|}{\sum_{k=1}^{m} a_{k}} \\
& +\frac{\mid \sum_{k=1}^{m} a_{k}\left(g\left(\tau_{k}, x^{*}\left(\phi_{1}\left(\tau_{k}\right)\right)\right) \int_{0}^{\tau_{k}} f\left(s, x\left(\phi_{2}(s)\right)\right) d s-g\left(\tau_{k}, x^{*}\left(\phi_{1}\left(\tau_{k}\right)\right)\right) \int_{0}^{\tau_{k}} f\left(s, x^{*}\left(\phi_{2}(s)\right)\right) d s \mid\right.}{\sum_{k=1}^{m} a_{k}} \\
& +\left|g\left(t, x\left(\phi_{1}(t)\right)\right) \int_{0}^{t} f\left(s, x\left(\phi_{2}(s)\right)\right) d s-g\left(t, x^{*}\left(\phi_{1}(t)\right)\right) \int_{0}^{t} f\left(s, x\left(\phi_{2}(s)\right)\right) d s\right| \\
& +\left|g\left(t, x^{*}\left(\phi_{1}(t)\right)\right) \int_{0}^{t} f\left(s, x\left(\phi_{2}(s)\right)\right) d s-g\left(t, x^{*}\left(\phi_{1}(t)\right)\right) \int_{0}^{t} f\left(s, x^{*}\left(\phi_{2}(s)\right)\right) d s\right| \\
& \leq \delta A \\
& +\frac{\sum_{k=1}^{m} a_{k} K\left\|x-x^{*}\right\|(K\|x\|+a)}{\sum_{k=1}^{m} a_{k}} \\
& +\frac{\sum_{k=1}^{m} a_{k} K\left\|x-x^{*}\right\|\left(K\left\|x^{*}\right\|+a\right)}{\sum_{k=1}^{m} a_{k}} \\
& +K\left\|x-x^{*}\right\|(K\|x\|+a)+K\left\|x-x^{*}\right\|\left(K\left\|x^{*}\right\|+a\right) \\
& \leq \delta A \\
& +2 K\left\|x-x^{*}\right\|(K r+a)+2 K\left\|x-x^{*}\right\|(K r+a) \\
& +2 K\left\|x-x^{*}\right\|(K r+a)+2 K\left\|x-x^{*}\right\|(K r+a) \\
& \leq \delta A+\left(4 K^{2} r+4 a K\right)\left\|x-x^{*}\right\| \text {. }
\end{aligned}
$$

Then,

$$
\left\|x-x^{*}\right\| \leq \delta A\left(1-4 K^{2} r-4 a K\right)^{-1} \leq \epsilon
$$

Definition 3. The unique solution of the problem in Equations (2) and (5) depends continuously on the initial data $a_{k}$ if $\epsilon>0, \exists \delta>0$ such that

$$
\sum_{k=1}^{m}\left|a_{k}-a_{k}^{*}\right| \leq \delta \quad \Rightarrow\left\|x-x^{*}\right\| \leq \epsilon
$$


where $x^{*}$ is the unique solution of the integral equation

$$
\begin{aligned}
& x^{*}(t)=\frac{1}{\sum_{k=1}^{m} a_{k}^{*}}\left[x_{0}-\sum_{k=1}^{m} a_{k}^{*} g\left(\tau_{k}, x^{*}\left(\phi_{1}\left(\tau_{k}\right)\right)\right) \int_{0}^{\tau_{k}} f\left(s, x^{*}\left(\phi_{2}(s)\right)\right) d s\right] \\
& +g\left(t, x^{*}\left(\phi_{1}(t)\right)\right) \int_{0}^{t} f\left(s, x^{*}\left(\phi_{2}(s)\right)\right) d s .
\end{aligned}
$$

Theorem 5. Let the assumptions $\left(I^{*}\right)-\left(I I^{*}\right)$ be satisfied; then, the unique solution of (5) and (2) depends continuously on $\sum_{k=1}^{m} a_{k}$. Then,

\section{Proof.}

$$
\begin{aligned}
& \left|x(t)-x^{*}(t)\right|=\frac{1}{\sum_{k=1}^{m} a_{k}}\left[x_{0}-\sum_{k=1}^{m} a_{k} g\left(\tau_{k}, x\left(\phi_{1}\left(\tau_{k}\right)\right)\right) \int_{0}^{\tau_{k}} f\left(s, x\left(\phi_{2}(s)\right)\right) d s\right] \\
& \left.\left.+g\left(t, x\left(\phi_{1}(t)\right)\right)\right) \int_{0}^{t} f\left(s, x\left(\phi_{2}(s)\right)\right)\right) d s-\frac{1}{\sum_{k=1}^{m} a_{k}^{*}}\left[x_{0}\right. \\
& \left.\left.-\sum_{k=1}^{m} a_{k}^{*} g\left(t, x^{*}\left(\phi_{1}\left(\tau_{k}\right)\right)\right) \int_{0}^{\tau_{k}} f\left(s, x^{*}\left(\phi_{2}(s)\right)\right) d s\right]-g\left(t, x^{*}\left(\phi_{1}(t)\right)\right) \int_{0}^{t} f\left(s, x^{*}\left(\phi_{2}(s)\right)\right)\right) d s \mid \\
& \leq \frac{\left|x_{0}\right|\left|\sum_{k=1}^{m} a_{k}-\sum_{k=1}^{m} a^{*}\right|}{\sum_{k=1}^{m} a k-\sum_{k=1}^{m} a^{*}} \\
& +\left|\frac{g\left(\tau_{k}, x\left(\phi_{1}\left(\tau_{k}\right)\right)\right) \int_{0}^{\tau_{k}} f\left(s, x\left(\phi_{2}(s)\right)\right) d s}{\sum_{k=1}^{m} a_{k}}-\frac{\left.g\left(\tau_{k}\right), x^{*}\left(\phi_{1}\left(\tau_{k}\right)\right)\right) \int_{0}^{\tau_{k}} f\left(s, x^{*}\left(\phi_{2}(s)\right)\right) d s}{\sum_{k=1}^{m} a_{k}^{*}}\right| \\
& +\mid g\left(t, x\left(\phi_{1}(t)\right) \int_{0}^{t} f\left(s, x\left(\phi_{2}(s)\right)\right) d s-g\left(\tau_{k}, x^{*}\left(\phi_{1}(t)\right)\right) \int_{0}^{t} f\left(s, x^{*}\left(\phi_{2}(s)\right)\right) d s \mid\right. \\
& \leq \frac{\left|x_{0}\right| \delta}{\sum_{k=1}^{m} a_{k} \sum_{k=1}^{m} a_{k}^{*}} \\
& +\frac{\left.\sum_{k=1}^{m} a_{k} \sum_{k=1}^{m} a_{k}^{*} \mid g\left(\tau_{k}, x\left(\phi_{1}\left(\tau_{k}\right)\right)\right)-g\left(\tau_{k}\right), x^{*}\left(\phi_{1}\left(\tau_{k}\right)\right)\right)\left|\int_{0}^{\tau_{k}}\right| f\left(s, x\left(\phi_{2}(s)\right)\right) \mid d s}{\sum_{k=1}^{m} a_{k} \sum_{k=1}^{m} a_{k}^{*}} \\
& +\frac{\sum_{k=1}^{m} a_{k} \sum_{k=1}^{m} a_{k}^{*}\left|g\left(\tau_{k}, x^{*}\left(\phi_{1}\left(\tau_{k}\right)\right)\right)\right| \int_{0}^{\tau_{k}}\left|f\left(s, x\left(\phi_{2}(s)\right)\right)-f\left(s, x^{*}\left(\phi_{2}(s)\right)\right)\right| d s}{\sum_{k=1}^{m} a_{k} \sum_{k=1}^{m} a_{k}^{*}} \\
& +\left|g\left(t, x\left(\phi_{1}(t)\right)\right)-g\left(t, x^{*}\left(\phi_{1}(t)\right)\right)\right| \int_{0}^{t}\left|f\left(s, x\left(\phi_{2}(s)\right)\right)\right| d s \\
& +\left|g\left(t, x^{*}\left(\phi_{1}(t)\right)\right)\right| \int_{0}^{t}\left|f\left(s, x\left(\phi_{2}(s)\right)\right)-f\left(s, x^{*}\left(\phi_{2}(s)\right)\right)\right| d s \\
& \leq\left|x_{0}\right| \delta A A^{*} \\
& +K\left\|x-x^{*}\right\|(K\|x\|+a) \\
& +K\left\|x-x^{*}\right\|\left(K\left\|x^{*}\right\|+a\right) \\
& +K\left\|x-x^{*}\right\|(K\|x\|+a)+K\left\|x-x^{*}\right\|\left(K\left\|x^{*}\right\|+a\right) \\
& \leq\left|x_{0}\right| \delta A A^{*}+\left(4 K^{2} r+4 a K\right)\left\|x-x^{*}\right\| . \\
&
\end{aligned}
$$

Then,

$$
\left\|x-x^{*}\right\| \leq\left|x_{0}\right| \delta A A^{*}\left(1-4 K^{2} r-4 a K\right)^{-1} \leq \epsilon .
$$

\section{Riemann-Stieltjes Integral Condition}

Let $x \in C[0,1]$ be the solution of the nonlocal boundary value problem in Equations (2) and (5). 
Let $a_{k}=\left(h\left(t_{k}\right)-h\left(t_{k-1}\right)\right)$, where $h$ is an increasing function, $\tau_{k} \in\left(t_{k-1}-t_{k}\right)$, and $0=t_{0}<t_{1}<t_{2}, \ldots<t_{m}=1$; then, the nonlocal condition (2) is

$$
\sum_{k=1}^{m}\left(h\left(t_{k}\right)-h\left(t_{k-1}\right)\right) x\left(\tau_{k}\right)=x_{0}
$$

and the limit implies

$$
\lim _{m \rightarrow \infty} \sum_{k=1}^{m}\left(h\left(t_{k}\right)-h\left(t_{k-1}\right)\right) x\left(\tau_{k}\right)=\int_{0}^{1} x(s) d h(s)=x_{0} .
$$

Theorem 6. Let the assumptions (I)-(III) be satisfied; then, the nonlocal boundary value problem of (5) and (3) has at least one solution given by

$$
\begin{aligned}
& \left.x(t)=\frac{1}{h(1)-h(0)}\left[x_{0}-\int_{0}^{1} g\left(s, x\left(\phi_{1}(s)\right)\right) \int_{0}^{s} f\left(\theta, x\left(\phi_{2}(\theta)\right)\right)\right) d \theta d h(s)\right] \\
& +g\left(t, x\left(\phi_{1}(t)\right)\right) \int_{0}^{t} f\left(s, x\left(\phi_{2}(s)\right)\right) d s .
\end{aligned}
$$

Proof. Let $h:[0,1] \rightarrow[0,1]$, and let $a_{k}=h\left(t_{k}\right)-h\left(t_{k-1}\right)$ be an increasing function. Then, the solution of (5) and (3) is given by

$$
\begin{aligned}
& x(t)=(h(1)-h(0))^{-1} x_{0} \\
& -(h(1)-h(0))^{-1} \sum_{k=1}^{m}\left(h\left(\tau_{k}\right)-h\left(\tau_{k-1}\right)\right) g\left(\tau_{k}, x\left(\phi_{1}\left(\tau_{k}\right)\right)\right) \int_{0}^{\tau_{k}} f\left(s, x\left(\phi_{2}(s)\right)\right) d s \\
& +g\left(t, x\left(\phi_{1}(t)\right)\right) \int_{0}^{t} f\left(s, x\left(\phi_{2}(s)\right)\right) d s .
\end{aligned}
$$

As $m \rightarrow \infty$, we obtain

$$
\begin{aligned}
& \lim _{m \rightarrow \infty} x(t)=(h(1)-h(0))^{-1} x_{0} \\
& -(h(1)-h(0))^{-1} \lim _{m \rightarrow \infty} \sum_{k=1}^{m}\left(h\left(\tau_{k}\right)-h\left(\tau_{k-1}\right)\right) g\left(\tau_{k}, x\left(\phi_{1}\left(\tau_{k}\right)\right)\right) \int_{0}^{\tau_{k}} f\left(s, x\left(\phi_{2}(s)\right)\right) d s \\
& +g\left(t, x\left(\phi_{1}(t)\right)\right) \int_{0}^{t} f\left(s, x\left(\phi_{2}(s)\right)\right) d s . \\
& \frac{1}{h(1)-h(0)}\left[x_{0}-\int_{0}^{1} g\left(s, x\left(\phi_{1}(s)\right)\right) \int_{0}^{s} f\left(\theta, x\left(\phi_{2}(\theta)\right)\right) d \theta d h(s)\right] \\
& +g\left(t, x\left(\phi_{1}(t)\right)\right) \int_{0}^{t} f\left(s, x\left(\phi_{2}(s)\right)\right) d s .
\end{aligned}
$$

\section{Infinite-Point Boundary Condition}

Theorem 7. Let the assumptions (I)-(III) be satisfied; then, the nonlocal boundary value problem of (5) and (4) has at least one solution given by

$$
\begin{aligned}
& x(t)=\frac{1}{\sum_{k=1}^{\infty} a_{k}}\left[x_{0}-\sum_{k=1}^{\infty} a_{k} g\left(\tau_{k}, x\left(\phi_{1}\left(\tau_{k}\right)\right)\right) \int_{0}^{\tau_{k}} f\left(s, x\left(\phi_{2}(s)\right)\right) d s\right] \\
& +g\left(t, x\left(\phi_{1}(t)\right)\right) \int_{0}^{t} f\left(s, x\left(\phi_{2}(s)\right)\right) d s .
\end{aligned}
$$


Proof. Let the assumptions of Theorem 1 be satisfied. Let $\sum_{k=1}^{m} a_{k}$ be convergent; then, take the limit of (5). We have

$$
\begin{array}{r}
\lim _{m \rightarrow \infty} x(t)=\lim _{m \rightarrow \infty} \frac{1}{\sum_{k=1}^{m} a_{k}}\left[x_{0}-\lim _{m \rightarrow \infty} \sum_{k=1}^{m} a_{k} g\left(\tau_{k}, x\left(\phi_{1}\left(\tau_{k}\right)\right)\right) \int_{0}^{\tau_{k}} f\left(s, x\left(\phi_{2}(s)\right)\right) d s\right] \\
+g\left(t, x\left(\phi_{1}(t)\right)\right) \int_{0}^{t} f\left(s, x\left(\phi_{2}(s)\right)\right) d s .
\end{array}
$$

Now,

$$
\begin{aligned}
& \left|a_{k} g\left(\tau_{k}, x\left(\phi_{1}\left(\tau_{k}\right)\right)\right)\right| \leq K\left|a_{k}\right| x\left(\phi_{1}\left(\tau_{k}\right)\right) \mid, \\
& \leq K\left|a_{k}\right|\|x\| \leq K\left|a_{k}\right| r .
\end{aligned}
$$

Then, by a comparison test, the series $\sum_{k=1}^{\infty} a_{k} g\left(\tau_{k}, x\left(\phi_{1}\left(\tau_{k}\right)\right)\right)$ is convergent and

$$
\begin{aligned}
& x(t)=\frac{1}{\sum_{k=1}^{\infty} a_{k}}\left[x_{0}-\sum_{k=1}^{\infty} a_{k} g\left(\tau_{k}, x\left(\phi_{1}\left(\tau_{k}\right)\right)\right) \int_{0}^{\tau_{k}} f\left(s, x\left(\phi_{2}(s)\right)\right) d s\right] \\
& +g\left(t, x\left(\phi_{1}(t)\right)\right) \int_{0}^{t} f\left(s, x\left(\phi_{2}(s)\right)\right) d s .
\end{aligned}
$$

Furthermore, from (10), we have

$$
\begin{aligned}
& \sum_{k=1}^{\infty} a_{k} x(t)=\sum_{k=1}^{\infty} a_{k} \frac{1}{\sum_{k=1}^{\infty} a_{k}}\left[x_{0}-\sum_{k=1}^{\infty} a_{k} g\left(\tau_{k}, x\left(\phi_{1}\left(\tau_{k}\right)\right)\right) \int_{0}^{\tau_{k}} f\left(s, x\left(\phi_{2}(s)\right)\right) d s\right] \\
& +g\left(\tau_{k}, x\left(\phi_{1}\left(\tau_{k}\right)\right)\right) \int_{0}^{\tau_{k}} f\left(s, x\left(\phi_{2}(s)\right)\right) d s=x_{0} .
\end{aligned}
$$

Example 1. Consider the boundary value problem, with nonlocal

$$
\frac{d}{d t}\left(\frac{x(t)-x(0)}{\frac{1}{4}\left(\frac{\cos (t)}{t+1}+x(t)\right)}\right)=\frac{1}{4}\left(t^{2}-t+e^{t} x(t)\right),
$$

with the nonlocal boundary condition

$$
\sum_{k=1}^{m}\left[\frac{1}{k}-\frac{1}{k+1}\right] x\left(\tau_{k}\right)=x_{0}, a_{k}>0 \tau_{k} \in[0,1]
$$

putting

$$
\begin{aligned}
& f(t, x(t))=\frac{1}{4}\left(t^{2}-2 t+e^{t} x(t)\right) \\
& g(t, x(t))=\frac{1}{4}\left(\frac{\cos (t)}{t+1}+x(t)\right)
\end{aligned}
$$

and

$$
|g(t, x(t))-g(t, y(t))| \leq \frac{1}{4}|x(t)-y(t)|
$$

then,

$$
\begin{aligned}
& \left|\frac{1}{4}\left(\frac{\cos (t)}{t+1}+x(t)\right)\right| \leq \frac{1}{4}\left|\left(\frac{1}{t+1}+x(t)\right)\right| \\
& \left|\frac{1}{4}\left(t^{2}-2 t+e^{t} x(t)\right)\right| \leq \frac{1}{4} \mid\left(t^{2}-t+x(t)\right)
\end{aligned}
$$


It is clear that assumptions (I)-(II) of Theorem 1 are satisfied with $a_{1}(t)=\frac{1}{4}\left|\frac{1}{t+1}\right| \in L^{1}[0,1]$; if $t=0$, then $a_{1}(0)=\frac{1}{4}$; if $t=1$, then $a_{1}(1)=0.12375$ and $a_{2}(t)=\frac{1}{4}\left|t^{2}\right| \in L^{1}[0,1] ;$ if $t=0$, then $a_{2}(0)=0$; and if $t=1$, then $a_{2}(1)=0$.

Then, $a=\sup \left\{0, \frac{1}{4}\right\}=\frac{1}{4}, K=\sup \left\{\frac{1}{4}, \frac{1}{4}\right\}=\frac{1}{4}, x_{0}=1$, and $\sum_{k=1}^{m} a_{k}=1$; then, $A\left|x_{0}\right|+$ $2(K r+a)^{2}-r=0=A\left|x_{0}\right|+2 K^{2} r^{2}+4 k a r+2 a^{2}-r=0$.

By applying Theorem 1, the nonlocal problem in Equations (10) and (11), has a continuous solution.

Example 2. Consider the boundary value problem with nonlocal

$$
\frac{d}{d t}\left(\frac{x(t)-x(0)}{\frac{1+2 t}{20}+e^{-t} \frac{1}{30}+\sqrt{\frac{2}{3}} x(t)}\right)=\frac{1}{7} \ln \left(\frac{20}{1+t}\right)+\frac{1}{3} x(t),
$$

with the nonlocal boundary condition (11).

Set

$$
\begin{aligned}
& f(t, y)=\frac{1}{7} \ln \left(\frac{20}{1+t}\right)+\frac{1}{3} y \\
& g(t, x)=\frac{1+2 t}{20}+e^{-t} \frac{1}{30}+\sqrt{\frac{2}{3}} x .
\end{aligned}
$$

We can easily deduce the following:

$$
\text { and } \quad \begin{aligned}
|f(t, x)-f(s, y)| & \leq \frac{1}{3}|x-y| \\
|g(t, x)-g(s, y)| & \leq \sqrt{\frac{2}{3}}|x(t)-y(t)| .
\end{aligned}
$$

Easily, we can verify the existence of a unique solution of the problem in Equations (11) and (12).

\section{Conclusions}

In this work, we proved the existence of at least one solution $x \in C[0,1]$ and its maximum and minimum of the nonlocal problem for the boundary value problem of hybrid functional differential inclusion (1)

$$
\frac{d}{d t}\left(\frac{x(t)-x(0)}{g\left(t, x\left(\phi_{1}(t)\right)\right)}\right) \in F\left(t, x\left(\phi_{2}(t)\right)\right), \quad t \in(0,1)
$$

with the nonlocal boundary condition (2)

$$
\sum_{k=1}^{m} a_{k} x\left(\tau_{k}\right)=x_{0}, a_{k}>0 \tau_{k} \in[0,1] .
$$

The uniqueness of the solution $x \in C[0,1]$ of the nonlocal problem for the boundary value problem of hybrid functional differential Equation (5)

$$
\frac{d}{d t}\left(\frac{x(t)-x(0)}{g\left(t, x\left(\phi_{1}(t)\right)\right)}\right)=f\left(t, x\left(\phi_{2}(t)\right)\right), \quad t \in(0,1)
$$

and its continuous dependence on $x_{0}$ and initial data $a_{k}$ is proven.

The results have been generalized for problems with the nonlocal conditions (3) and (4).

Author Contributions: Conceptualization, A.M.A.E.-S., W.G.E.-S. and S.S.A. All authors have read and agreed to the published version of the manuscript.

Funding: This research received no external funding. 
Institutional Review Board Statement: Not applicable.

Informed Consent Statement: Not applicable.

Data Availability Statement: Not applicable.

Acknowledgments: Authors are grateful to referees for their useful comments and remarks that helped to improve this work.

Conflicts of Interest: The authors declare no conflict of interest.

\section{References}

1. Dhage, B.C. Quadratic perturbations of periodic boundary value problems of second order ordinary differential equations. Differ. Equ. Appl. 2010, 2, 465-486. [CrossRef]

2. Dhage, B.C. Basic results in the theory of hybrid differential equations with linear perturbations of second type. Tamkang J. Math. 2013, 44, 171-186. [CrossRef]

3. El-Sayed, A.M.A.; Ibrahim, A.G. Set-valued integral equation of fractional orders. Appl. Math. Comput. 2001, 118, 113-121. [CrossRef]

4. El-Sayed, A.M.A.; Bin-Taher, E.O.A. Nonlocal problem for a multi-term fractional order differential equation. J. Nath. Anal. 2001, 29, 1445-1451.

5. Herzallah, M.A.E.; Baleanu, D. On fractional order hybrid differential equations. Abstr. Appl. Anal. 2014, 2014, 389386. [CrossRef]

6. El-Sayed, A.M.A.; Ahmed, R.G. Existence of solutions for a functional integro-differential equation with infinite point and integral conditions. Int. J. Appl. Comput. Math. 2019, 5, 1-15. [CrossRef]

7. Ahmad, B.; Ntouyas, J.T. A nonlocal hybrid boundary value problem of Caputo fractional integro-differential equations. Acta Math. Sci. 2016, 36, 1631-1640. [CrossRef]

8. Dhage, B.C. Basic results in the theory of hybrid differential equations with mixed perturbations of second type. Funct. Differ. Equ. 2012, 19, 87-106.

9. Deimling, K. Multi-Valued Differential Equations; de Gruyter: Berlin, Germany, 1992.

10. El-Sayed, A.M.A.; Ibrahim, A.G. Multivalued fractional differential equations. Appl. Math. Comput. 1995, 68, 15-50. [CrossRef]

11. Fierro, R.; Martines, R.C.; Morales, C.H. Caratheodory selections for multi-valued mappings. Nonlinear Anal. 2006, 64, 1229-1235. [CrossRef]

12. Hashem, H.H.G.; Alsehail, H.M.H. Qualitative aspects of the fractional Air-Borne diseases model with Mittage-Leffler kernel. Adv. Math. Sci. J. 2021, 10, 1-15. [CrossRef]

13. Hashem, H.H.G.; Alrashidi, H.O. Characteristics of solutions of nonlinear neutral integrodifferential equation via Chandrasekhar integral. J. Math. Comput. Sci. 2021, 24, 173-185. [CrossRef]

14. Seba, D. Nonlinear fractional differential inclusion with nonlocal fractional integro-differential boundary conditions in Banach spaces. Math. Bohem. 2017, 142, 309-321. [CrossRef]

15. El-Sayed, A.M.A.; Ahmed, R.G. Infinite point and Riemann-Stieltjes integral conditions for an integro-differential equation. Nonlinear Anal. Modelling Control 2019, 24, 733-754. [CrossRef]

16. El-Sayed, A.M.A.; Ahmed, R.G. Solvability of a coupled system of functional integro-differential equations with infinite point and Riemann-Stieltjes integral conditions. Appl. Math. Comput. 2020, 370, 1-15. [CrossRef]

17. Melliani, S.; Hilal, K.; Hannabou, M. Existence results in the theory of hybrid fractional integro-differential equations. J. Univers. Math. 2018, 1, 166-179.

18. Srivastava, H.M.; El-Sayed, A.M.A.; Gaafar, F.M. A Class of Nonlinear Boundary Value Problems for an Arbitrary Fractional-Order Differential Equation with the Riemann-Stieltjes Functional Integral and Infinite-Point Boundary Conditions. Symmetry 2018, 10, 508. [CrossRef]

19. Boucherif, A.; Precup, R. On the nonlocal initial value problem for first order differential equations. Fixed Point Theory 2003, 4 , 205-212.

20. Curtain, R.F.; Pritchard, A.J. Functional Analysis in Modern Applied Mathematics; Academic Press: Cambridge, MA, USA, 1977.

21. Dhage, B.C. On a-condensing mappings in Banach algebras. Math. Stud. 1994, 63, 146-152. 